\title{
A combination of temperature, vegetation indexes and albedo, as obtained by airborne hyperspectral remote sensing, for the evaluation of soil moisture
}

\author{
Jean-Claude Krapez* and Albert Olioso** \\ * ONERA, The French Aerospace Lab \\ 13661 Salon de Provence, France \\ krapez@onera.fr \\ **INRA, UMR 1114 EMMAH (INRA - UAPV), \\ 84914 Avignon Cedex 9, France \\ olioso@avignon.inra.fr
}

\begin{abstract}
Temperature as obtained by infrared remote sensing of bare soil or vegetation covered soil during day depends on soil water content ranging from the surface down to the root-zone. The main reason is that evaporation and plant transpiration cools down the soil surface and the leaves. However surface temperature does not only depend on soil moisture. It has to be combined with other measured variables. In this work we start with the classical combination between temperature and a vegetation index and we explore the benefit of adding the albedo or the cellulose absorption index to retrieve the surface soil moisture. The correlation between the inferred Soil Vegetation Wetness Index and the true moisture content is analyzed based on the thermal infrared and visible-NIR hyperspectral images recorded during Hymap 2007 campaign over Camargue (France).

KEY WORDS: soil moisture, soil wetness, remote sensing, triangle method, vegetation index, hyperspectral, thermal infrared
\end{abstract}




\section{Introduction}

Information on soil moisture is of first importance for irrigation scheduling and for improving crop yield, but also in water management in the climate change context and for the evaluation of the anthropic ecosystem impact. Remote sensing with earth observation instruments has been considered for a few decades to provide the requested information over large areas and at various scales.

Vegetation and soil temperatures have long been recognized as an indicator of water availability. As a matter of fact, under proper water supply, the transpiration cools the leaves towards air temperature. Water is exchanged through the stomata, i.e. the pores in the leaf epidermis. On the opposite, under water stress condition, the plant closes its stomata to limit transpiration. This leads to foliage temperature increase. Similarly, the soil surface temperature is inversely correlated with water content due to evaporation. Additionally, soil drying induces a reduction of soil thermal inertia which also leads to soil temperature increase.

Thermal sensors used for environment monitoring on board of satellite or airplane have a spatial resolution ranging from a few meters to a couple of kilometres. The consequence is that in the footprint corresponding to a pixel, the surface is seldom homogenous: most pixels are a mixture of vegetation and soil. The radiance reaching the elementary detector is thus a combination of the radiance coming from leaves and from soil. As a consequence, the brightness temperature depends on temperature and emissivity of both soil and vegetation, and on the vegetation cover fraction. Part for this reason, the problem of evaluating the soil moisture is underdetermined when only considering the brightness temperature: a same brightness temperature value can be associated with a large range of soil moisture. It is thus necessary to add other observation data to reduce this underdetermination and finally to reduce the uncertainty of the inferred moisture.

A method proposed a few years ago consists in jointly analyzing the temperature and a vegetation index which is used as a proxy of the vegetation cover. Such index could be the classical NDVI (Normalized Difference Vegetation Index) which corresponds to the normalized difference between the signals recorded in the red and in the near infrared (typically at about $0.65 \mu \mathrm{m}$ and $0.8 \mu \mathrm{m}$ ). This index is indeed strongly correlated with the vegetation cover: it ranges from about 0.1 for bare soil to values close to 0.8 for dense and green vegetation, depending on the wavelength choice. It is also correlated with foliage density which is expressed by LAI, Leaf Area Index (total surface of leaves per unit surface). However NDVI is also sensitive to the soil spectral characteristics. Other indexes were proposed for being less dependant on soil background (Rondeaux et al., 1996). Among them we considered TSAVI (Transformed Soil Adjusted Vegetation Index) in this work (its definition is given later in Eq. [3]). Other vegetation indexes designed for providing a proxy for vegetation cover could also be used. For this reason we will denote VI for the chosen vegetation index. 
When plotting the two-dimensional distribution of temperature and vegetation index (T-VI) corresponding to an area with well distributed vegetation cover and moisture content, one gets a triangular of trapezoidal cloud of points (see Fig. 1). The four vertices correspond to the extreme conditions of a crop: the bottom vertices (low VI) correspond to dry and saturated bare soil, whereas the upper vertices (High VI) correspond to well-irrigated and water-stressed full cover vegetation (Moran et al., 1994). The left limit is called the "wet" (or "cold") edge whereas the right limit is called the "dry" (or "warm") edge. It is usually admitted that the soil dryness linearly increases when moving from the left ("wet") edge to the right ("dry") edge. For this reason, a soil moisture index was defined from the relative distance of a given point to the dry edge (a constant VI). Similarly, it is argued that the vegetation stress increases from the "wet" edge to the "dry" edge. A consequence is that the position of a particular point relatively to these extreme lines could also be a measure of the evapotranspiration flux at the time of the remote sensing test.

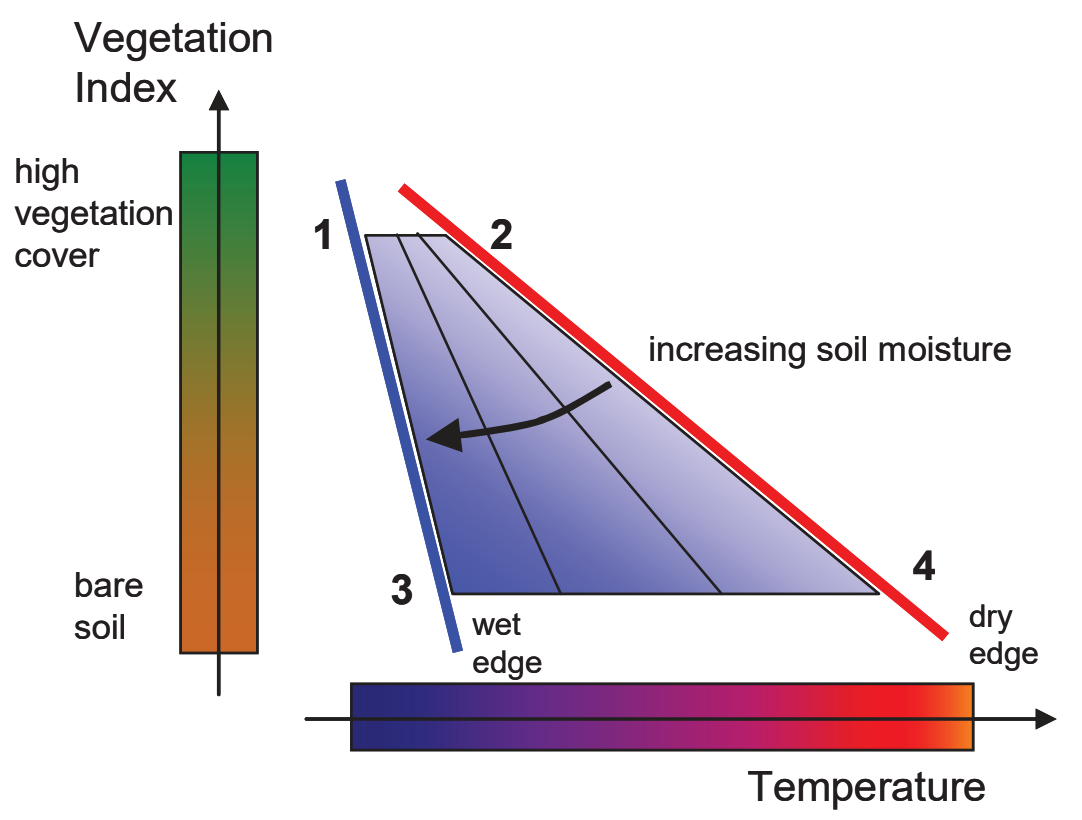

Figure 1. Principle of soil moisture mapping from a Temperature-Vegetation Index (T-VI) plot. The vertices of the trapezoidal distribution correspond to: (1) wellwatered full cover, (2) non-transpiring full cover, (3) wet bare soil, (4) dry bare soil. Soil moisture increases from the dry edge (on the right) to the wet edge (on the left) (Moran et al., 1994).

Different methods were devised for defining the position of the wet and dry edges. The empirical method uses the experimental T-VI distribution and linear edges are set to fit its border (Sandholt et al., 2002). Iso-moisture straight lines are then distributed linearly between the wet edge and the dry edge. Another method is based on the use of Penman-Monteith equation for evapotranspiration (which is derived from an energy budget equation) for expressing the difference between surface temperature and air temperature for the four extreme cases that define the vertices of the trapezoidal T-VI 
plot (Moran et al., 1994). As before, iso-moisture straight lines are distributed linearly between the wet edge and the dry edge. Still another method is based on the use of a SVAT (Soil Vegetation Atmosphere Transfer) model in order to evaluate the radiation flux, the convective flux and the water/vapor fluxes between soil, vegetation and atmosphere (Carlson et al., 1995). The SVAT model allows building iso-moisture lines as well as iso-evaporation lines to which the T-VI distribution can then be compared. After inversion, one retrieves a moisture map of the overflown area.

These methods were compared in (Krapez et al., 2009) where some improvements were also proposed. We highlighted the possibility to infer not only a map of the surface moisture but also an estimation of the root-zone moisture.

Nevertheless the concept of exploiting only T-VI (eventually together with some meteorological data like in the methods described in (Moran et al., 1994) and (Carlson et al., 1995)) is based on a simple representation of the thermal and radiative properties of the complex soil/vegetation structure and of the exchanges therein. However, a large number of parameters, in addition to soil moisture, are influencing the apparent soil/canopy temperature. Furthermore these parameters may vary between the different vegetation types and the different soil types that are present in the scene. Spreading the temperature distribution along only one dimension like the VI is not enough for efficiently separating the sensitivities and for allowing an unambiguous determination of soil moisture. In addition to temperature and vegetation index, the authors in (Chauhan et al., 2003) suggested to consider surface albedo as retrieved from visible-near IR reflectance signals. As a matter of fact the albedo $\alpha$ has a high influence on the net radiation flux and as such has a significant impact on temperature. Furthermore, with nowadays hyperspectral instruments, it is an easy task to evaluate with a good precision this optical property. In (Chauhan et al., 2003) the identification process was divided in two phases. The first phase is a learning phase which consists in a fitting procedure between the optical remote sensing data $T, N D V I, \alpha$ on one hand and Mo on the other hand, where Mo is the reference moisture inferred from low-resolution microwave remote sensing. The regression law is a polynomial limited to second order terms:

$$
\begin{aligned}
M o= & a_{000}+a_{001} \alpha^{*}+a_{010} T^{*}+a_{100} N D V I^{*} \\
& +a_{002} \alpha^{* 2}+a_{020} T^{* 2}+a_{200} N D V I^{* 2} \\
& +a_{011} \alpha^{*} T^{*}+a_{101} \alpha^{*} N D V I^{*}+a_{110} T^{*} N D V I^{*}
\end{aligned}
$$

Each observable variable $T^{*}, N D V I^{*}, \alpha^{*}$ is obtained first by downscaling the optical data to the microwave resolution and then by normalizing it with the corresponding minimum and maximum values. High resolution moisture maps were then constructed by applying the quadratic relation in Eq. [1] to the raw, i.e. high resolution, optical images. 
We propose to take into account the albedo in a different way which avoids referring to microwave data for the learning process. The idea is merely to extent the procedure defined for 2D T-VI plots in (Sandholt et al., 2002) to 3D T-VI- $\alpha$ plots. Dry and wet edges now become dry and wet 2D surfaces in the 3D representation. We define the Soil-Vegetation Wetness Index (SVWI) from the relative distance between the considered pixel and the dry surface.

When introducing the albedo, it is expected to better separate areas according to the absorbed solar radiation. We also looked for separating vegetated areas according to the fraction of green vegetation versus senescent vegetation. A suitable index for quantifying the fraction of senescent vegetation is CAI (Cellulose Absorption Index). As a matter of fact, a broad absorption band near $2.1 \mu \mathrm{m}$ appears in all compounds possessing alcoholic -OH groups such as sugars, starch and cellulose. This absorption band appears in the reflectance spectra of dry plant residues and a cellulose absorption index (CAI) based on the reflectance in three bands - two on the shoulders at $2 \mu \mathrm{m}$ and $2.2 \mu \mathrm{m}$ and one at $2.1 \mu \mathrm{m}$ (cellulose-lignin absorption maximum) was proposed in (Daughtry, 2001):

$$
C A I=0.5\left(\rho_{2.0}+\rho_{2.2}-2 \rho_{2.1}\right)
$$

where $\rho_{i}$ is the reflectance at wavelength $i$.

A linear unmixing approach using Normalized Difference Vegetation Index (NDVI) and the Cellulose Absorption Index (CAI) proved indeed to be efficient in providing an estimation of fractional cover of photosynthetic vegetation (fPV), nonphotosynthetic vegetation (fNPV) and bare soil (Guerschman et al., 2009).

In the same manner as before with albedo, we propose to use CAI as an additional observation parameter and to exploit the three-dimensional T-VI-CAI distribution features to infer a soil moisture map.

The aim of this study is to analyse the benefit of adding a third index to the classical T-VI empirical method for soil moisture mapping (Sandholt, 2002). The proposed procedures will be applied on remote sensing data obtained during the HyEurope 2007 campaign over Camargue (France) with HyMap and ATM sensors operated by DLR. HyMap is a hyperspectral imager with 128 bands from $0.45 \mu \mathrm{m}$ to $2.5 \mu \mathrm{m}$ whereas ATM is a multispectral imager with 11 bands from $0.42 \mu \mathrm{m}$ to $13 \mu \mathrm{m}$.

\section{Measurement campaign}

\subsection{Airborne remote sensing campaign}

During the HyEurope 2007 campaign, a HyMap hyperspectral sensor (HyVista Corporation Website, 2011) was operated by DLR, Oberpfaffenhofen, Germany, on board of a Do228 airplane over several sites in Europe (DLR HyEurope Campain, 2010). Seventeen flight lines were flown on June $23^{\text {rd }}$ over Camargue region, south of France. 
HyMap is a spectroradiometer utilizing diffraction gratings and four 32-element detector arrays (1 Si, 3 liquid nitrogen-cooled $\mathrm{InSb})$. It measures radiation in 128 spectral bands between $0.45 \mu \mathrm{m}$ and $2.48 \mu \mathrm{m}$. It is a whisk-broom type scanner, i.e. a mirror scans across the airplane path, reflecting light towards the four arrays which collect a spectrum one pixel at a time. The angular width is $61.3^{\circ}$ with 512 pixels in each line ( $2 \mathrm{mrad}$ across track IFOV and $2.5 \mathrm{mrad}$ along track IFOV). At the $1950 \mathrm{~m}$ mean flight altitude the swath was thus $2.3 \mathrm{~km}$ and the resolution was at nadir about $4 \mathrm{~m} \times 5 \mathrm{~m}$.

Together with HyMap was flown the multispectral sensor Daedalus AADS 1268 "Airborne Thematic Mapper" (ATM). It is also a whisk-broom type scanner. Measurement is performed in ten bands from $0.42 \mu \mathrm{m}$ to $2.23 \mu \mathrm{m}$ plus in one large thermal infrared band from 8 to $13 \mu \mathrm{m}$. The angular width is $86^{\circ}$ with 716 pixels in a line. The swath was thus $3.6 \mathrm{~km}$ and the nadir pixel dimension of approximately $5 \mathrm{~m}$.

Data from HyMap and ATM were orthorectified and resampled at $4 \mathrm{~m} \times 4 \mathrm{~m}$ resolution by DLR. Fluctuations of altitude were taken into account in the geometric correction. An atmospheric correction was also applied to HyMap data, but not to ATM data. The ATM thermal channel was nevertheless calibrated absolutely in temperature units (brightness temperature).

Channel $16(0.675 \mu \mathrm{m})$ and $26(0.819 \mu \mathrm{m})$ from HyMap were combined to provide a vegetation index map of the flown area. A spectrum recorded over bare soil was used to evaluate the soil line coefficients $a, b$ which are necessary for computing TSAVI (Transformed Soil Adjusted Vegetation Index) (Rondeaux et al., 1996).

$$
T S A V I=a\left(\rho_{0.82}-a \rho_{0.67}-b\right) /\left[\rho_{0.67}-a\left(\rho_{0.82}-b\right)+0.08\left(1+a^{2}\right)\right]
$$

Channel $11(8-13 \mu \mathrm{m})$ from ATM provided the brightness temperature map. Modtran4 simulations for atmospheric radiation and transmission were performed at ONERA in order to convert at-sensor brightness temperature to on-ground true temperature as described in (Lagouarde et al., 2000). For this purpose an estimation of the effective emissivity map was necessary. Effective emissivity was computed from a weighted average of soil emissivity and vegetation emissivity according to the model described in (Valor and Caselles, 1996) which takes into account mean values for vegetation emissivity and for bare soil emissivity. Vegetation emissivity was taken from (Coll et al., 2003) whereas mean soil emissivity was evaluated from measurements performed on saturated and dry soil samples taken from six different fields in flight line 2 (Lesaignoux et al., 2009). Standard deviation of altitude fluctuations was $3.7 \mathrm{~m}$. Its impact on soil temperature evaluation was estimated to be less than $0.02 \mathrm{~K}$; this correction was thus discarded.

For present analysis we focused on flight line $\mathrm{n}^{\circ} 2$ which was flown at $10 \mathrm{~h} 20$ UTC over a region with crops of wheat, alfalfa (flowering plant cultivated as forage crop), meadows, rice and vineyards and some bare soil fields (average coordinates: $\mathrm{N}$ $43.629^{\circ} \mathrm{E} 4.477^{\circ}$ ). 


\subsection{In-situ measurements}

In-situ measurements were performed by INRA in a series of fields in the central part of flight line $n^{\circ} 2$. In particular, a series of soil samples were extracted in the 0 $5 \mathrm{~cm}$ upper soil layer on June $25^{\text {th }}$ for gravimetric water content measurement ( 5 samples per spot, 3 spots per field or per crop type) (see Table 1).

In the following we will also focus the discussion on three other types of crops: harvested wheat, tilled wheat and rice (see Table 2).

\begin{tabular}{|c|c|c|}
\hline 2000 & 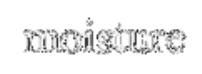 & 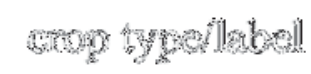 \\
\hline 1 & 996 & nowadowa \\
\hline 2 & 92.8 & Whe \\
\hline 3 & 22,2 & renderom \\
\hline 4 & $1 / 4.9$ & silfith \\
\hline 5 & 7.6 & (4) \\
\hline 6 & $\sqrt{6}$ & gilf \\
\hline 7 & 13.2 & Whom A \\
\hline 8 & 24.9 & Whord \\
\hline 9 & 13.0 & whothere \\
\hline
\end{tabular}

\begin{tabular}{|c|c|c|}
\hline 20000 i & bonolistive & 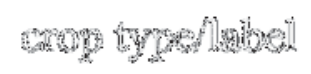 \\
\hline 10 & 52.46 & baresoil \\
\hline il & 9.3 & 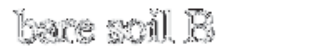 \\
\hline 12 & $9 A$ & 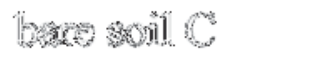 \\
\hline 13 & 74 & donghthod stid A \\
\hline 1 息 & 7.8 & plonghos sold I \\
\hline 15 & 8.8 & plonglod god d \\
\hline 16 & 10,4 & 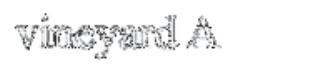 \\
\hline 87 & 102 & 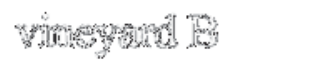 \\
\hline 13 & 9.4 & 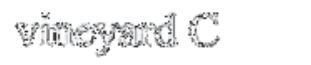 \\
\hline
\end{tabular}

Table 1. List of in situ water content gravimetric measurements and mean moisture value (\% weight).

\begin{tabular}{|c|c|}
\hline motom & Wp the \\
\hline 19 & 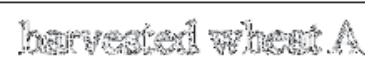 \\
\hline 20 & 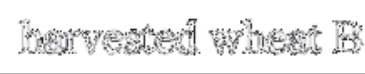 \\
\hline $2 \pi$ & 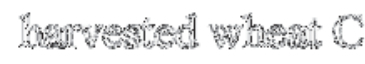 \\
\hline 28 & 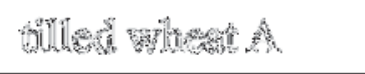 \\
\hline 23 & 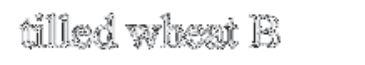 \\
\hline 264 & thiles wherito \\
\hline 24 & 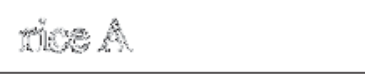 \\
\hline 26 & nire \\
\hline 27 & trikns \\
\hline
\end{tabular}

Table 2. Additional fields without in situ moisture measurement. 


\section{Temperature and Vegetation Index maps}

Temperature map as obtained with Daedalus ATM sensor is reported in Fig. 2 left. Corresponding TSAVI (Transformed Soil Adjusted Vegetation Index) map as obtained with Hymap sensor is reported in Fig. 2 right. The color scale was chosen to represent high vegetation cover in green and bare soil in yellow. In general, the highest temperature values are observed over bare soils. Especially cold plots correspond to irrigated rice fields.
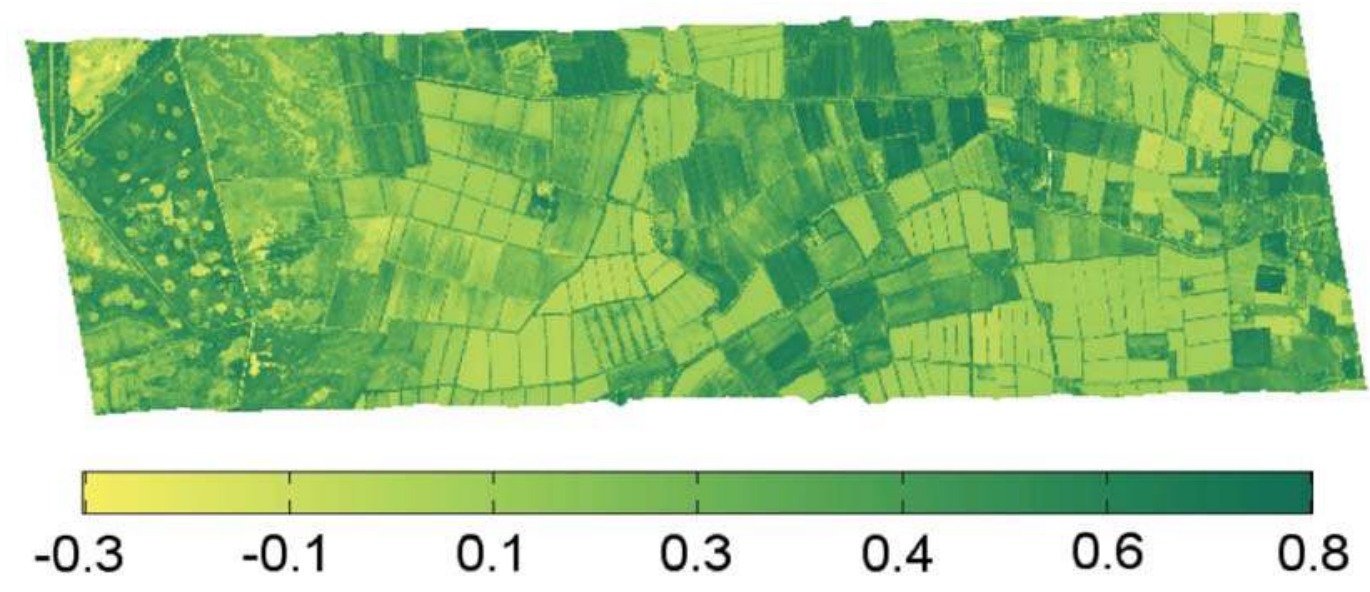

Transformed soil adjusted vegetation index (TSAVI)

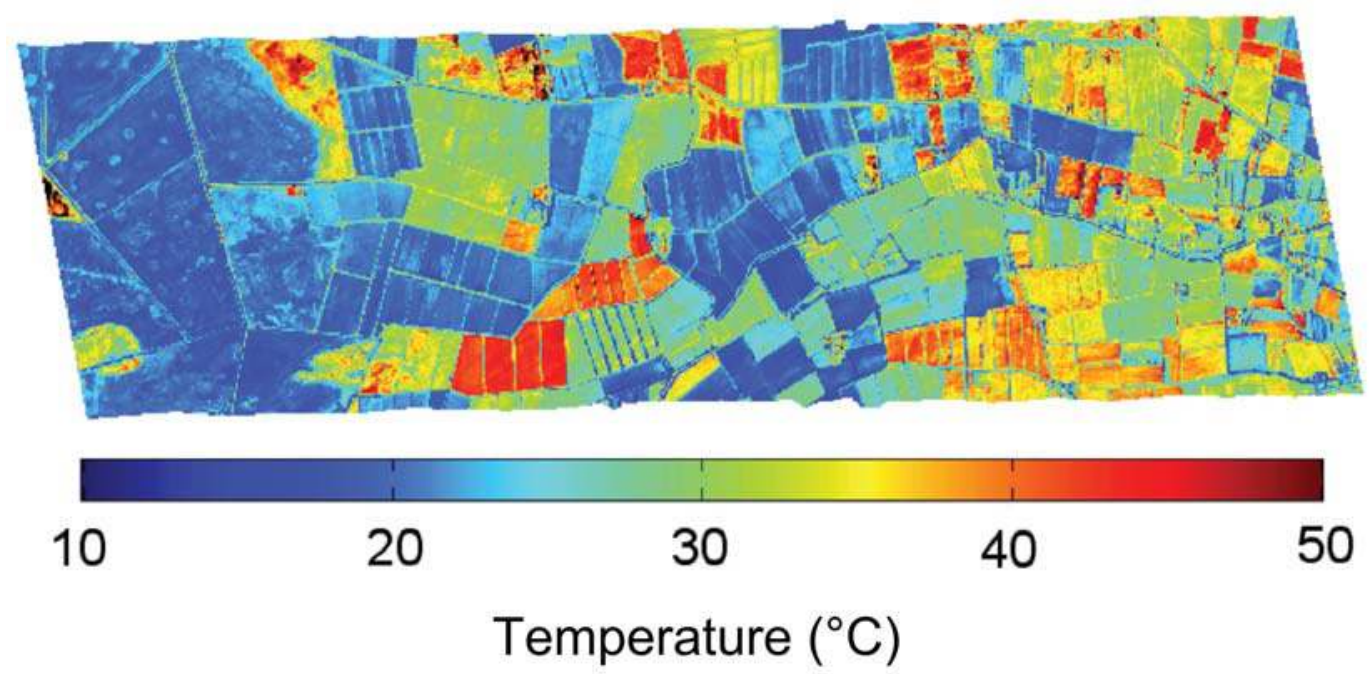

Figure 2. Map of vegetation index TSAVI (left) and of temperature (right) recorded over flight line 2 (about $2.5 \mathrm{~km} \times 8.3 \mathrm{~km}$ ).

\section{Moisture identification from 2D T-VI distribution}

When plotting the Temperature-Vegetation Index data for whole flight line, one gets the distribution in Fig. 3. It is roughly trapezoidal with an apex at high vegetation index (high vegetation cover). The drawing of the straight lines which define the wet and dry edges as described in (Sandholt et al., 2002) is rather subjective. Here we 
chose a more objective approach based on statistics: for each TSAVI interval of width 0.05 , a low temperature limit and a high temperature limit are defined from the $1.5 \%$ and the $98.5 \%$ percentile. The results are then interpolated to provide the blue and red curves, respectively wet edge and dry edge in Fig. 3. By this way the distribution is well bounded; in the same time, possible outliers are rejected.

At each Vegetation Index level, the moisture index is defined as the relative distance to the dry edge. We call it Soil Vegetation Wetness Index (SVWI). Except for very few points, it ranges between 0 and 1 .

$$
S V W I_{i j}=\frac{T_{i j}-T_{d r y}\left(T S A V I_{i j}\right)}{T_{w e t}\left(T S A V I_{i j}\right)-T_{d r y}\left(T S A V I_{i j}\right)}
$$

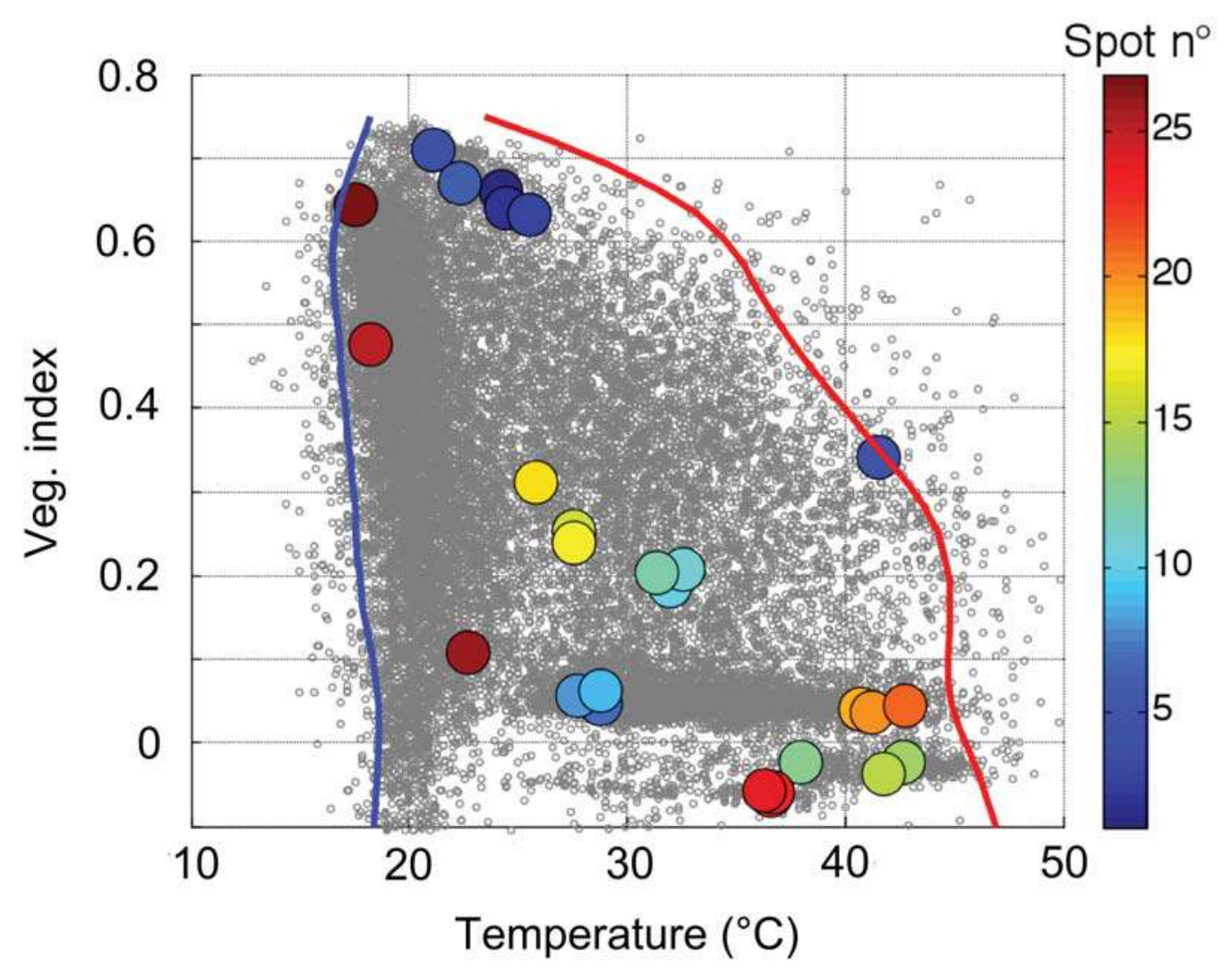

Figure 3. Temperature-Vegetation Index plot with the location of wet edge (in blue) and the dry edge (in red). The results for the 27 spots listed in Table 1 and 2 are represented with dots with scaled color.

In Fig. 4 this moisture index is compared to the measured moisture (in \% weight) for the 18 spots listed in Table 1 . The dots are not arranged along a straight line. This imperfect correlation has several explanations:

- The results for meadow fields are those which are farthest from the regression line (the three dark blue dots in the upper part). Meadow spots actually present high TSAVI values and it is well known that temperature shows a decreasing sensitivity to 
soil water content for increasing values of Leaf Area Index (i.e. at the top of the T-VI distribution). Another possible reason is that high VI areas in flight line 2 may offer a too narrow range of soil moisture (the dry edge would be underestimated at the top of the trapezoid); in such situation the present empirical approach for moisture evaluation would lead to a biased wetness index.

- In-situ measured moisture is in \% weight whereas SVWI is linked to volume water content: indeed meadow soil has generally a lower density than bare soil.

- In-situ measurements were performed two days after the remote sensing campaign.

Anyway, when excluding the meadow data, the correlation between SVWI and gravimetric moisture is reasonably good: the correlation coefficient $R^{2}$ reaches 0.61 .

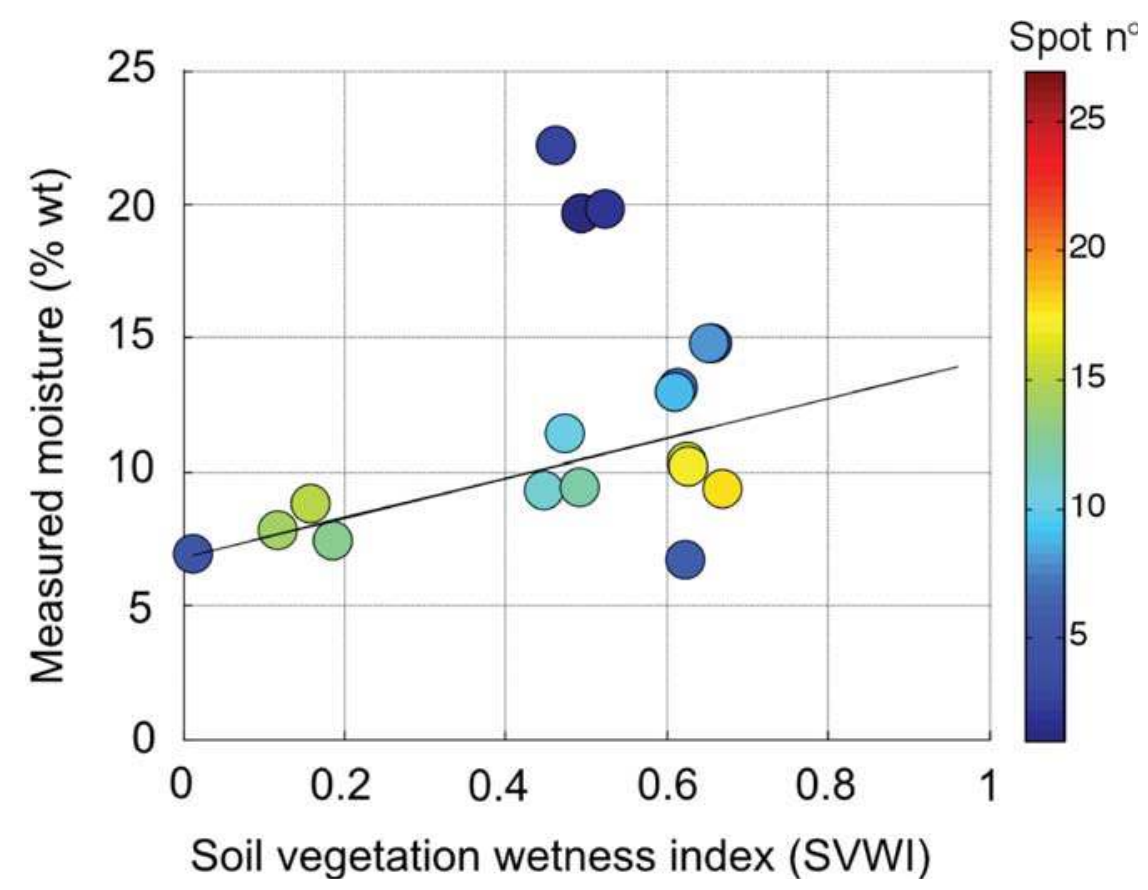

Figure 4. Comparison between the Soil Vegetation Wetness Index (SVWI) computed from remote sensing data and the in situ measured moisture. SVWI was obtained by using the 2D T-VI distribution in Fig. 3.

\section{Moisture identification from 3D distributions T-VI- $\alpha$ or T-VI-CAI}

When considering the albedo or the Cellulose Absorption Index (CAI) as an additional observable parameter, one gets the 3D distributions reported in Fig. 5. When adding a third parameter, the wet edge and the dry edge become bounding surfaces. 
Similarly to the previous case, we defined by computing the $1.5 \%$ temperature percentile and the $98.5 \%$ temperature percentile values over cells obtained after discretizing the VI- $\alpha$ plane, respectively the VI-CAI plane.
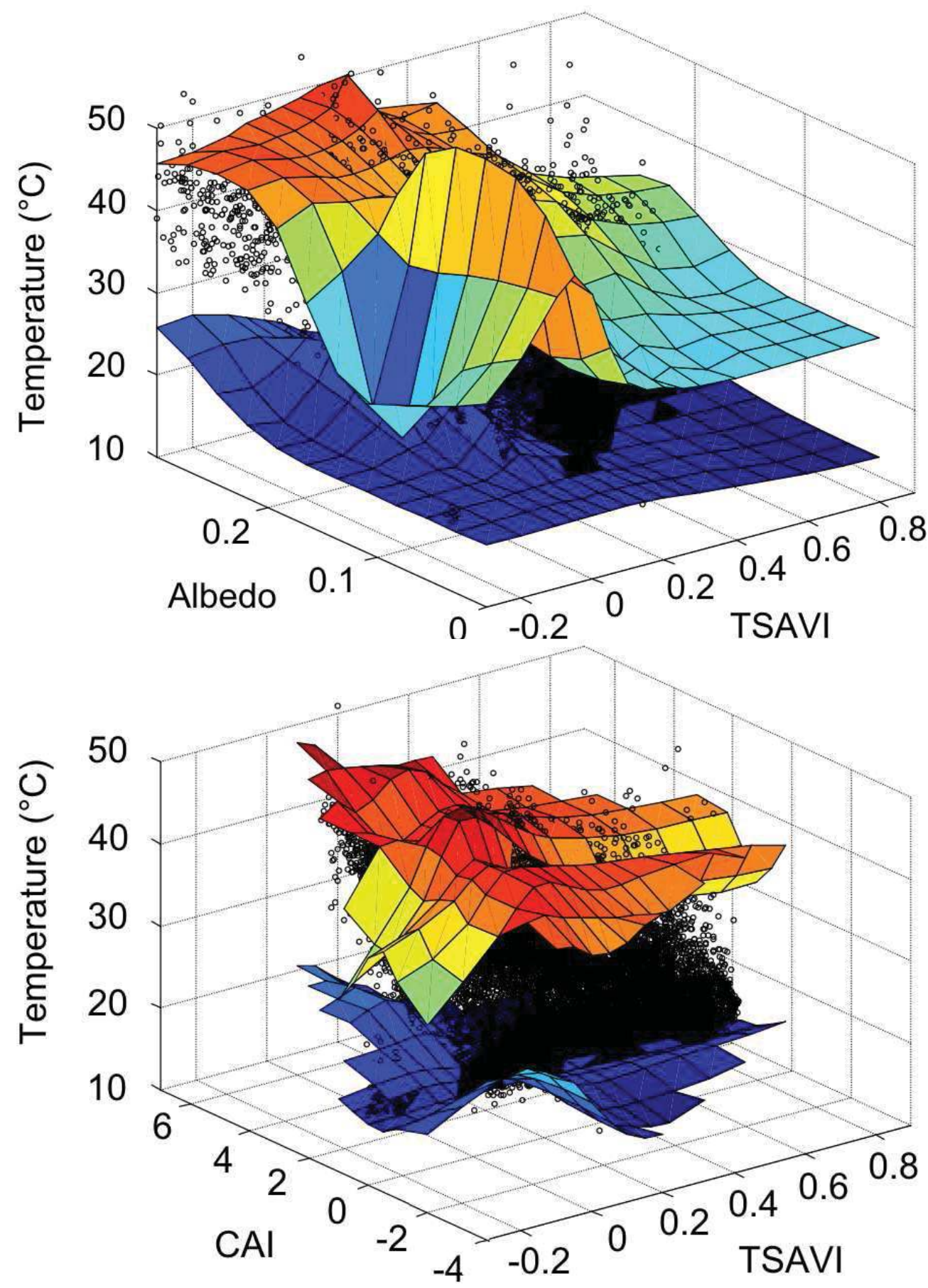

Figure 5. Distribution of temperature, Vegetation Index and either albedo (top) or Cellulose Absorption Index (bottom). The "wet" surface and the "dry" surface are defined to stick to the distributions from bellow and from top.

The correlation coefficient $\mathrm{R}^{2}$ between SVWI and gravimetric moisture content slightly diminishes to 0.57 when using albedo whereas it increases to 0.69 when using CAI (see Fig. 6). Therefore introducing the albedo doesn't seem to provide a significant improvement, at least for present types of crops. The use of the Cellulose Absorption 
Index (CAI) seems to be more promising. This may be due to the fact that this index has the ability to separate senescent vegetation, like ripe wheat and harvested wheat, from bare soils and from green vegetation. Different classes of surfaces which present highly different processes regarding evapo-transpiration but which previously merged in the classical T-VI plot, are now separated in the T-VI-CAI plot.

The proposed Wetness Index map as obtained from the analysis of the T-VI-CAI distribution is plotted in Fig. 7. A strong correlation is found between the wetness index and the crop type: with only a few exceptions, the rice fields are the wettest, and the driest soil is observed in alfalfa fields and ploughed fields. For wheat fields, soil moisture decreases when going from ripe vegetation field, to tilled field and then to harvested field. Bare soils and tilled wheat fields present similar wetness index.

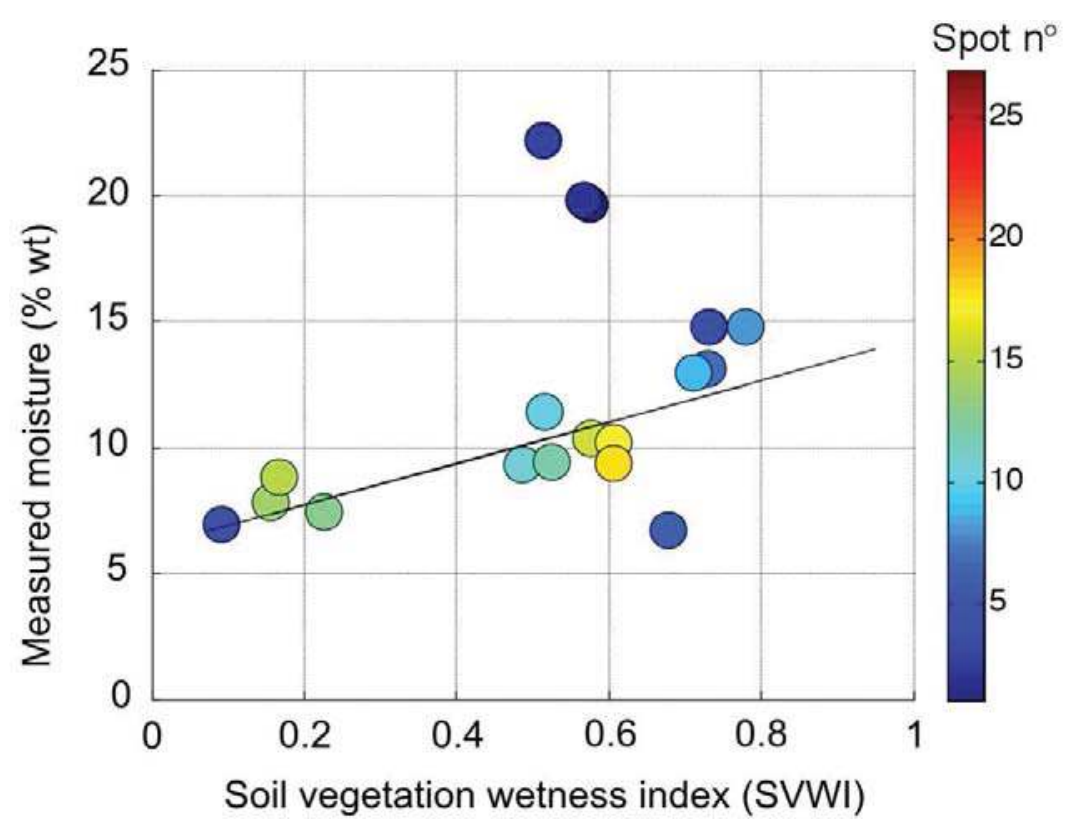

Figure 6. Comparison between the Soil Vegetation Wetness Index (SVWI) computed from remote sensing data and the in situ measured moisture. SVWI was obtained by using the T-VI-CAI distribution in fig. 5-bottom.

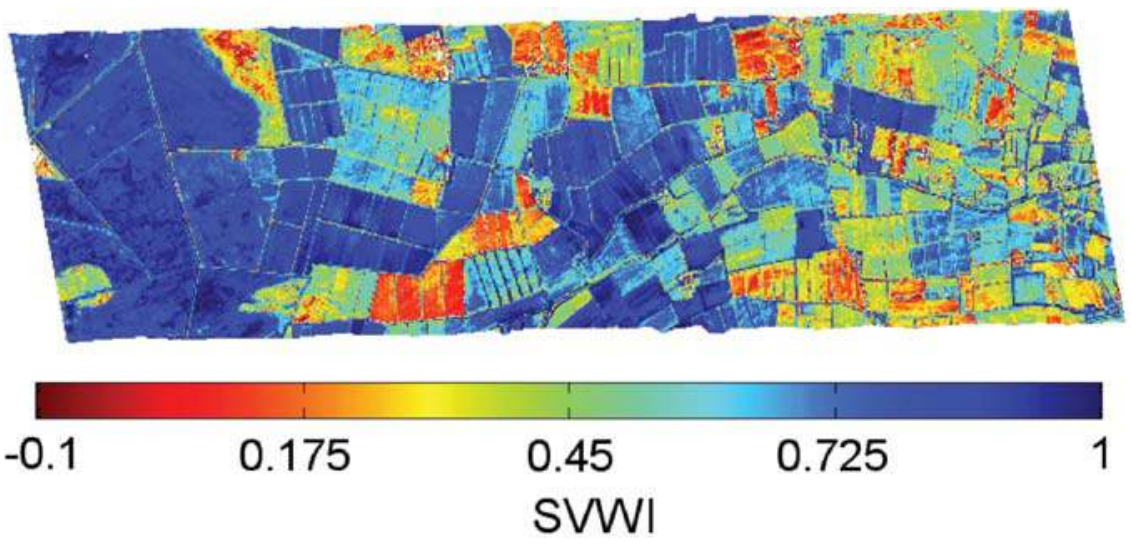

Figure 7. Soil Vegetation Wetness Index (SVWI) map computed from remote sensing data by exploiting the T-VI-CAI distribution in fig. 5-right (high water content is represented in blue). 


\section{Conclusion}

Adding a third observation parameter like CAI or albedo to temperature and vegetation index which are used in the classical "triangle method" can bring the advantage of discriminating areas having different moisture content but otherwise presenting the same T-VI values. A better correlation is thus expected between the calculated moisture and the measured one. For the analyzed crops, the addition of CAI proved to be efficient, contrarily to albedo. The number of reference data used for the correlation analysis was however limited. Other remote sensing databases should be considered to further assess the benefit of the proposed method.

\section{Acknowledgements}

The authors are indebted to DLR, Oberpfaffenhofen, Germany, for providing the HyEurope 2007 remote sensing data. The authors would like to thank INRA team for ground truth measurements, A. Lesaignoux and S. Fabre from ONERA for providing the soil spectra and the French Programme National de Télédétection Spatiale (INSU - PNTS) for providing funding for the acquisition of ground truth data in Camargue. This research is within the framework of ONERA Enviro project.

\section{References}

Carlson, T.N., Gillies, R.R., Schmugge, T. G., "An interpretation of methodologies for indirect measurement of soil water content", Agricultural and Forest Meteorology, 77, 191-205, 1995.

Chauhan N.S., Miller S., Ardanuy P., "Spaceborne soil moisture estimation at high resolution: a microwave-optical/IR synergistic approach", Int. J. Remote Sensing, vol. 24(22), pp. 45994622, 2003.

Coll C., Caselles V., Valor E., Rubio E., "Validation of temperature-emissivity separation and split-window methods from TIMS data and ground measurements", Remote Sensing of Environment, 85, 232-242, 2003.

Daughtry C.S.T., "Discriminating Crop Residues from Soil by Shortwave Infrared Reflectance", Agronomy J., 93:125-131, 2001.

DLR HyEurope Campain, 2010: http://www.opairs.aero/hymap_cmp en.html

Guerschman J.P., Hill M.J., Renzullo L.J., Barrett D.J., Marks A.S., Botha E.J., "Estimating fractional cover of photosynthetic vegetation, non-photosynthetic vegetation and bare soil in the Australian tropical savanna region upscaling the EO-1 Hyperion and MODIS sensors", Remote Sensing of Environment, 113, 928-945, 2009.

HyVista Corporation Website, 2011: http://www.hyvista.com/?page id=275.

Krapez J.-C., Olioso A., Coudert B., "Comparison of three methods based on the temperatureNDVI diagram for soil moisture characterization", Remote Sensing For Agriculture, 
Ecosystems, and Hydrology XI, Berlin 31 August - 3 Sept., ed. by Christopher M. U. Neale, Antonino Maltese, Proc. of SPIE, vol. 7472, 74720y 1-12, 2009.

Lagouarde J.-C., Ballans H., Moreau P., Guyon D., Coaboeuf D., "Experimental Study of Brightness Surface Temperature Angular Variations of Maritime Pine (Pinus pinaster) Stands", Remote Sensing of Environment, 72, 17-34, 2000.

Lesaignoux A., Fabre S., Briottet X., Olioso A., "Influence of surface soil moisture on spectral reflectance of bare soil in the 0.4 - $15 \mu \mathrm{m}$ domain", EARSeL'09, Tel Aviv, Israel, 2009, online proceedings: http://www.earsel.org/workshops/IS Tel-Aviv_2009/PDF/earselPROCEEDINGS/3024\%20Lesaignoux.pdf

Moran, M.S., Clarke, T.R., Inoue, Y., Vidal, A., "Estimating Crop water deficit using the relation between surface-air temperature and spectral vegetation index", Remote Sensing of Environment, vol. 49, 246-263, 1994.

Rondeaux G., Steven M., Baret F., "Optimization of soil adjusted vegetation indices”, Remote Sensing of Environment, vol. 55, 95-107, 1996.

Sandholt, I., Rasmussen, K., Andersen, J., "A simple interpretation of the surface temperaturevegetation index space for assessment of surface moisture status", Remote Sensing of Environment, 79, 213-224, 2002.

Valor E. and Caselles V., "Mapping Land Surface Emissivity from NDVI: Application to European, African, and South American Areas", Remote Sensing of Environment, 57:167184, 1996. 